\title{
Anne Réach-Ngô
}

\section{Accéder à la matière du livre Le détour du fac-similé}

Avec les premiers développements de l'édition numérique, et notamment l'apparition, ces dernières années, des tablettes, liseuses et autres supports technologiques de lecture, l'imaginaire du livre imprimé semble avoir été ébranlé dans ce qui faisait son identité même : le texte se trouve dissocié de son support originel, le livre, pour devenir un ensemble de données que l'on peut incarner en diverses formes, suivant les besoins du moment. Dans le même temps toutefois, et sans doute en réaction au spectre de la dématérialisation de l'écrit, les fac-similés connaissent une véritable vogue éditoriale, venant renouveler une tradition ancrée de longue date.

Le fac-similé, défini par Pascal Griener comme un «document cognitif ${ }^{1}$ », relève de ressorts sémiotiques complexes qui témoignent de l'ambivalence de la relation que l'époque contemporaine entretient avec la matérialité du livre ancien. Entendu suivant son sens le plus étymologique, le fac-similé se rattache à sa manière aux arts d'imitation et d'adaptation qui rendent possible la transmission, de nos jours, des arts du passé. L'impératif latin fac simile, «fais semblablement», «fais à l'identique », évoque autant l'efficacité d'un faire, d'une «poiétique ", que la mise à distance, reconnue et désavouée à la fois, propre à la «simulation»: copier quasi à l'identique, ou du moins faire semblant d'être identique, suivant le jeu de l'illusion référentielle de toute littérature fictionnelle. Les éditions en fac-similé des œuvres anciennes relèventelles alors d'une forme de fétichisme bibliophilique à valeur commémorative, d'un goût et d'une curiosité intellectuelle pour les realia du passé, ou ne témoignent-elles pas également d'une actualité de la «matière du livre », et, partant, d'une nouvelle «manière de lire ", attentive à un engagement de tout le corps, progressivement écarté de la lecture par le développement des « écrits d'écran »?

Avant d'entreprendre un rapide tour d'horizon de la production de facsimilés sur le marché éditorial contemporain, il convient de rappeler que la

1 P. Griener, «La culture du fac-similé », La République de l'œil. L'expérience de l'art au siècle des Lumières, Paris, O. Jacob, 2010, p. 225-246. 
production actuelle de fac-similés s'inscrit dans une tradition qui remonte au XVII siècle, suivant des enjeux qu'Ève Netchine a bien résumés : une fonction d'authentification, le fac-similé ayant d'abord eu une valeur légale, signalant la copie conforme à l'original ; une fonction documentaire et testimoniale, le facsimilé constituant une "chalcographie paléographique» conservant l'image exacte des monuments historiques et littéraires de la France; une fonction pédagogique, le fac-similé participant à la diffusion des savoirs; et une fonction bibliophilique, le fac-similé visant à « offrir des recueils d'exemples et des corpus de modèles aux artistes et aux amateurs ${ }^{2} »$.

Ces différentes fonctions se retrouvent diversement dans les fac-similés publiés de nos jours, mais leur contexte de réception à l'aube du XXIe siècle, notamment avec l'essor des nouvelles «technologies de l'esprit ", contribue à en renouveler la valeur et la portée. C'est à la fois dans la relation que ces ouvrages entretiennent avec une tradition ancienne et en réaction aux mutations contemporaines de l'écrit que l'on s'intéressera au phénomène éditorial du facsimilé. La présentation des trois principaux types de fac-similés disponibles à l'heure actuelle (fac-similés de manuscrits, fac-similés d'éditions anciennes et impressions à la demande) prendra appui sur l'analyse des rhétoriques publicitaires mises en œuvre dans les brochures et sites internet des maisons d'édition qui les produisent et les diffusent. Elle s'attachera à mettre au jour les différents imaginaires que ces discours sollicitent, les usages du livre qu'ils rendent possible et l'accès à l'œuvre ancienne qu'ils favorisent.

\section{Fac-similés de manuscrits : restituer la matière de l'écrit, solliciter les sens du lecteur}

Le marché du fac-similé vise en premier lieu les amateurs de manuscrits anciens. Au-delà du prix prohibitif des originaux, la difficulté de consultation des livres manuscrits, réservée aux spécialistes autorisés, concourt au développement de luxueuses reproductions de manuscrits médiévaux, parfois serties de pierres véritables et enluminées à l'or fin, selon des techniques ancestrales. Tirées à moins d'un millier d'exemplaires numérotés, ces fac-similés sont destinés à un public de connaisseurs. Ils restituent l'objectif premier des manuscrits d'apparat qui leur ont servi de modèle et qui constituaient, au Moyen Âge, des œeuvres d'art à part entière ${ }^{3}$.

2 È. Netchine, «Faire voir, faire preuve et faire apprendre ou le fac-similé dans l'édition savante, littéraire et bibliophilique du XVII ${ }^{e}$ au XIX siècle ", dans les Actes du colloque Utopia instrumentalis: fac-similés au musée (Paris, Cité de la musique, novembre 2010), p. 34, disponible en ligne: http://www.citedelamusique.fr/pdf/note_programme/np_11054.pdf (consulté le 18 septembre 2016).

3 Nous renvoyons, à titre d'exemples, aux sites internet de ces différentes maisons d'édition : Facsimilé éditions («leader mondial de l'édition de fac-similés enluminés»); GM Éditeurs («maison d'édition consacrée à la reproduction et commercialisation de fac-similés de codex 
Les noms des maisons d'édition concernées (Patrimonio, Octavo, Quaternio, etc.), les discours publicitaires qui figurent dans leurs brochures et sur leurs sites, reposent sur un argument de vente emprunté au marché de la reproduction d'art. Il s'agit, tout en rendant accessible une œuvre d'art unique convertie en produit culturel, de participer à la conservation et à la valorisation du patrimoine. La qualité de la reproduction rend légitime la substitution du facsimilé à l'original, au point que ces éditions finissent par prendre la valeur d'un objet patrimonial. C'est du moins ce que déclare Manuel Moleiro sur le site de sa maison d'édition: "nous pouvons affirmer qu'un codex de M. Moleiro est plus qu'un simple fac-similé, c'est un "quasi-original" ». Susceptible de venir remplacer occasionnellement l'original, trop fragile ou absent de son institution d'origine, le fac-similé de grande qualité est ainsi à même de provoquer une émotion esthétique.

L'intérêt du grand public pour la culture du manuscrit ancien se manifeste également lors des nombreuses expositions consacrées aux enluminures, livres d'heures et autres œuvres célèbres du Moyen Âge, comme Le Roman de la Rose récemment exposé à la bibliothèque de l'Arsenal ${ }^{5}$. Les reproductions, dans les catalogues, de manuscrits médiévaux en fac-similé permettent de rendre ces ouvrages accessibles à un plus large public, tout comme le font certaines revues spécialisées comme Art de l'enluminure, qui publie des fac-similés complets ou partiels de grands manuscrits médiévaux. Il arrive parfois que ce type d'ouvrages devienne lui-même objet de collection. C'est le cas par exemple du catalogue de l'exposition du Louvre consacrée aux Très riches heures du duc de Berry en 2012, qui a été rapidement épuisé et fait désormais l'objet de spéculations sur internet, pour la revente d'occasion ${ }^{6}$.

Cet engouement pour les manuscrits reproduits en fac-similé se manifeste également chez les amateurs d'autographes, papiers de grands hommes et

médiévaux, livres de gravures, manuscrits et incunables, en tirages courts et limités à une seule édition de seulement 997 exemplaires destinée à des bibliophiles, collectionneurs et amants de l'art en général »); Octavo, qui se réclame de l'idéal humaniste de la Renaissance ("Our purpose is to use the best in advanced technology to uncover and enhance the seminal ideas of the past in ways that will provide inspiration for the present and future »; " Notre but est d'utiliser les technologies de pointe pour révéler et améliorer les idées pionnières du passé afin qu'elles inspirent le présent et l'avenir »); Quaternio, parvenant à allier « des éditions en fac-similé répondant aux critères les plus élevés de la reproduction à l'identique, des matériaux choisis avec soin et une fabrication irréprochable » et «une très grande flexibilité face aux souhaits de notre clientèle »; la maison d'édition catalane Manuel Moleiro ("Reliés en peau tannée suivant les méthodes d'autrefois et édités sur un papier spécial fabriqué à la main, nos codex reproduisent toutes les nuances des peintures, le parchemin, l'or, l'argent »), etc.

4 http://www.moleiro.com/fr/au-sujet-de-m-moleiro.htm (consulté le 12 avril 2013).

5 «L'art d'aimer au Moyen Âge : Le Roman de la rose », Bibliothèque de l'Arsenal (6 nov. 2012 17 févr. 2013). Citons également l'exposition du Musée de la Renaissance consacrée à "Geoffroy Tory, imprimeur de François I ${ }^{\mathrm{er}}$, graphiste avant la lettre » (6 avril-4 juillet 2011).

6 P. Torres-Guardiola et al., Les Belles Heures du duc de Berry, Paris, Louvre éditions / Somogy, 2012. 
brouillons d'auteurs, ce dont témoigne le succès renouvelé du Musée des Lettres et des Manuscrits, à Paris ${ }^{7}$. Nombreuses sont aussi les collections éditoriales à proposer des reproductions de manuscrits modernes en version intégrale, partielle ou sous forme d'anthologies. Tout comme dans les éditions scientifiques de manuscrits médiévaux en fac-similé, généralement précédées d'une préface d'un universitaire, et, suivant les collections, assortie d'une transcription et d'une annotation critique $^{8}$, ces éditions génétiques trouvent leur intérêt dans la reproduction de la matérialité même de l'écrit. La facture du manuscrit tout comme la disposition de la page d'écriture constituent une inscription du geste créateur de l'artiste, qu'il s'agisse de l'enlumineur comme du moine-copiste pour les manuscrits médiévaux ou de l'auteur rédigeant ses œuvres à la main pour les manuscrits modernes. La rhétorique publicitaire mise en œuvre par toutes ces collections atteste le souci de faire entrer le lecteur dans l'intimité de l'auteur, en exploitant le mythe romantique de l'écrivain au travail dans la solitude de son cabinet ${ }^{9}$.

La coloration patrimoniale dont jouit ce type de publications constitue un argument de vente très efficace. Aussi peut-on associer ce phénomène à la vogue des reproductions de documents historiques, ou encore de pièces et médailles ${ }^{10}$. Non plus pensé sur le mode de la reproduction d'art mais sur celui de l'archive, le fac-similé acquiert le statut de témoin, voire de trace du passé, quand bien même il n'en constituerait qu'une pâle reproduction. Les collections de « livres coffrets », dont on constate le succès, notamment en période de fêtes, mettent à disposition du grand public des documents d'époque constitués en dossier

7 Ce musée est également assez représentatif de la complexité des enjeux liés à l'acquisition de manuscrits et autographes, y compris dans leur dimension financière.

8 Nous pensons notamment aux éditions Champion ou à la Société des Textes Français Modernes.

9 Citons les éditions Textuel qui ont créé une série autour de la collection de lettres d'artistes célèbres, rassemblées par A.-M. Springer. La présentation qui en est faite est révélatrice de la démarche archivistique que recèle une telle entreprise et du souci d'authenticité des pièces présentées : «Donner à voir le geste initial, l'élan créateur ; motiver une rencontre avec le document brut, telle est l'ambition des éditions en fac-similés : manuscrits, brouillons, ébauches, ratures, biffures jalonnent et révèlent le cheminement et les affres de la genèse. Une porte entrouverte sur l'atelier des écrivains, des poètes, des peintres et des auteurscompositeurs ». De même, « L'Or du temps », dirigée par P.-M. de Biasi, est une collection de fac-similés littéraires ou artistiques qui «a pour ambition de révéler des textes souvent méconnus, dans leur aura de rareté, mais aussi de donner au lecteur la possibilité d'accéder à l'élan créateur premier à travers le mouvement original de l'écriture manuscrite ». Citons également la collection «Sources» proposée par les Puf, en association avec la Fondation Martin Bodmer à Genève qui abrite des «trésors du patrimoine littéraire mondial » ou encore la collection lancée en partenariat avec la BnF visant à rassembler des manuscrits d'auteurs classés par genres littéraires, parue chez R. Laffont : « Les plus beaux manuscrits... ».

10 Il est intéressant de constater que les éditions du «Trésor du Patrimoine » comprennent une collection consacrée à la numismatique, le « Club français de la Monnaie ». 
d'archives, le lecteur pouvant alors se prendre au jeu de l'exploration historique et au «mystère » des vieux papiers ${ }^{11}$.

Poussant plus loin la stratégie marketing, certaines maisons d'édition ne s'en tiennent pas à la transmission d'un ersatz de patrimoine mais procèdent ellesmêmes à l'acte de patrimonialisation : c'est le cas par exemple de la maison d'édition des Saint-Pères qui s'est donnée pour «mission », depuis 2012, de faire paraître une collection des manuscrits de dix auteurs vivants qui ont marqué le siècle, à commencer par Amélie Nothomb qui fait paraître, pour les vingt ans d'Hygiène de l'assassin, le manuscrit de l'œuvre en fac-similé, en un coffret de luxe tiré à un millier d'exemplaires, avant que ne paraisse à sa suite un manuscrit de Jean d'Ormesson. Présentée comme l'un des rares auteurs de nos jours à rédiger encore ses écrits à la main, Amélie Nothomb connaît alors, par le biais de la publication de sa première œuvre, une forme de canonisation qui l'inscrit dans la tradition des grands auteurs du passé. Une telle promotion de l'ouvrage tend à inscrire le fac-similé dans la catégorie des « beaux livres » tout en lui conférant le statut de «pièce collector ».

Si ces éditions ne concernent pas, majoritairement, les œuvres d'Ancien Régime, dont on n'a conservé globalement que peu de manuscrits et avanttextes, ces divers produits éditoriaux témoignent de l'existence d'un véritable marché de la reproduction imprimée. Les fac-similés viennent satisfaire un même attachement des amateurs de livres et de littérature - et ce ne sont pas nécessairement les mêmes - pour la mémoire matérielle des œuvres, associée à une quête d'authenticité et d'exotisme temporel. Cette "poétique du facsimilé ", qui s'est notamment construite autour de la reproduction des manuscrits, médiévaux et modernes, joue sans doute pour beaucoup dans le développement actuel de la reproduction des éditions anciennes, comme si la valeur du manuscrit, désormais considéré comme une pré-histoire du livre à l'heure de l'écrit d'écran, s'était étendue aux éditions imprimées d'œuvres anciennes.

\section{Le fac-similé d'éditions anciennes : de l'exotisme temporel à l'émotion plastique}

Bien que radicalement différents des fac-similés de manuscrits, les fac-similés d'éditions anciennes font intervenir des ressorts bibliophiliques assez similaires : ils répondent au désir d'avoir entre les mains, d'incorporer à sa bibliothèque

11 Soulignons la rhétorique du dévoilement et du secret exploitée dans la présentation du coffret consacré à Versailles qu'a réalisé P.-J. Rémy (Versailles, Paris, Gründ, "Histoire sur le vif», 2008) : «Pour nous, il en dévoile les secrets : les secrets politiques comme les secrets d'alcôve. Ce récit passionnant est accompagné de 40 fac-similés de documents d'époque et de plus de 200 photographies, qui permettent de revivre l'Histoire de façon réellement dynamique, et font de ce livre-objet un véritable musée à domicile ». 
l'objet-livre dont on apprécie la facture, l'écrit que l'on prend plaisir à lire, afin de trouver dans la matérialité du livre une autre forme d'intimité avec l'œuvre. Deux cas méritent toutefois d'être distingués.

Certaines éditions en fac-similé reproduisent des ouvrages dont la facture matérielle participe de l'identité de l'œuvre et ce, pour diverses raisons. La publication elle-même peut apparaitre comme un événement dans le processus de réalisation de l'œuvre, de l'édition princeps à l'édition annotée, ce dont rendent compte, par exemple, les nombreuses reproductions en fac-similé de l'«Exemplaire de Bordeaux» des Essais de Montaigne ${ }^{12}$. Dans un tel cas, l'édition-source s'apparente à un manuscrit par la rareté du document reproduit.

L'œuvre peut aussi se caractériser par son hybridité, alliant le texte et l'image : c'est le cas des livres d'emblèmes qui ont particulièrement marqué la création littéraire à la Renaissance, tel le livre d'Alciat dont l'édition de 1551 est parue chez Slatkine Reprints en 199713. On rejoint ici la reproduction en facsimilé de bandes dessinées anciennes, qui connaissent un grand succès de nos jours, reproduisant des œuvres qui ont marqué leur temps comme les aventures de Tintin ou de Bécassine. La présence d'illustrations de qualité justifie également l'édition, comme pour les Fables de La Fontaine illustrées par Gustave Doré ou par Benjamin Rabier ${ }^{14}$. La reproduction en fac-similé est également pratique courante dans certaines maisons d'édition spécialisées dans les ouvrages scientifiques (médecine, botanique, architecture...), telles les Éditions de Boccard ou Jacques Gabbay. Dans ce type d'ouvrages, les schémas et images ont une valeur argumentative et composent le corps même de l'œuvre: les illustrations tiennent lieu de réceptacle des savoirs. Certains éditeurs exploitent alors ce créneau à destination du grand public, comme les éditions Gutenberg Reprints pour les ouvrages d'ésotérisme : la possession du livre, sa manipulation font partie de l'univers de représentation et des valeurs du monde de l'alchimie.

Dans toutes ces éditions, la matérialité du livre relève de l'identité même de l'œuvre, soit parce qu'elle a été pensée par le même auteur que celui du texte, soit parce que celui qui a mis en livre ces textes a signé son travail et que la facture de l'ouvrage est exceptionnelle, ou encore parce que la matérialité du livre participe spécifiquement d'un rituel où trouve place le dispositif de communication de l'œuvre, comme dans le cas des ouvrages d'ésotérisme. Mais n'est-ce pas le cas de toute œuvre littéraire? La dimension esthétique,

12 Citons à titre d'exemple : Essais de Montaigne : exemplaire de Bordeaux : reproduction en quadrichromie avec notes manuscrites marginales, éd. établie et présentée par Ph. Desan, Paris, Classiques Garnier, 2011.

13 Emblèmes d'André Alciat, «fac-similé de l'édition lyonnaise Macé-Bonhomme de 1551 », préfacée par P. Laurens, Paris, Klincksieck, 1997.

14 Voir les Fables de La Fontaine illustrées par Benjamin Rabier, fac-similé de l'édition de Paris, J. Tallandier, 1906, Paris, Go multimédia, 2012. Plus propre à la culture typographique d'Ancien Régime : G. Tory, Champ fleury, Art et science de la vraie proportion des lettres, préf. de PaulMarie Grinevald, Paris, Bibliothèque de l'image, 1998. 
l'intervention de l'artiste ou du compositeur, le projet éditorial qui a présidé à la conception du livre méritent reproduction en soi. Texte et livre ne faisant qu'un, donner à lire l'œuvre nécessite de donner accès à la matérialité du livre qui participe de son identité.

Aussi rencontre-t-on, au-delà de la reproduction de ces éditions exceptionnelles, des fac-similés de ce que l'on pourrait appeler des «imprimés ordinaires ». Il s'agit de reproduction d'œuvres anciennes pour lesquelles la mise en livre ne présente aucune particularité remarquable par rapport aux productions de l'époque ${ }^{15}$. Ces reproductions en fac-similé, destinées aux curieux et amateurs de livres anciens désireux de posséder des copies d'éditions anciennes à plus faible coût que leurs originaux, peut apparaître comme un remède à ce que le bibliographe et bibliothécaire anglais du XIXe siècle, Thomas Frognall Dibdin, a appelé «the Bibliomania or Book Madness ${ }^{16}$ ». Le succès, encore de nos jours, de la collection des Bibliolâtres de France datant des années 1940 témoigne de ce goût pour la matérialité du livre ancien, l'ouvrage de belle facture, sur de grandes feuilles pliées, non coupées et reliées, respectant les caractéristiques propres à la présentation des œuvres anciennes.

Proposant un produit plus acessible financièrement, certaines maisons d'édition exploitent les réseaux de distribution habituels pour rendre disponibles leurs fac-similés en librairie, à des prix comparables à ceux des publications contemporaines. C'est le cas notamment de la collection Fac Simil, diffusée par l'entreprise Maxtor. Les ouvrages, thermocollés et convertis en formats standardisés, reproduisent le texte dans sa mise en page d'époque. Toutefois, par souci d'homégénisation et afin de satisfaire le plaisir de l'œil contemporain, sensible à l'appel des images, la charte graphique de la collection, réutilisée pour toutes les périodes sans différenciation, a intégré sur la page de couverture une illustration en couleur sur un fond comme jauni par le temps, sans tenir compte sur ce point de l'édition originale. Si ce type de réaménagement relève d'une forme d'infidélité à l'œuvre initiale, il constitue également un bon moyen de renvoyer au lecteur un imaginaire du livre ancien qui lui parle sans doute plus que la réalité même de l'objet reproduit.

La pratique de la réimpression d'éditions anciennes constitue enfin, de nos jours, la marque de fabrique de certaines maisons d'édition, telle Slatkine qui se définit comme un « réimprimeur de textes rares»et dispose d'une collection importante :

15 Cet intérêt pour les éditions anciennes en elles-mêmes, dans ce qu'elles véhiculent d'un rapport au livre passé, se manifeste très nettement au Salon du livre ancien et de la bibliophilie.

16 The Bibliomania or Book Madness, containing some account of the history, symptoms, and cure of this fatal disease, Londres, 1809. C'est ce même désir bibliomaniaque qui avait concouru à l'importante production, au $\mathrm{XIX}^{\mathrm{e}}$ siècle, de réimpressions de textes anciens et rares datant du $\mathrm{XV}^{\mathrm{e}}$ et $\mathrm{XVI}{ }^{e}$ siècles et reproduits en des éditions soignées, tirées en un petit nombre d'exemplaires sur du beau papier, avec parfois un ou deux exemplaires sur vélin, et que les amateurs rendaient ensuite uniques en les faisant relier suivant leurs goûts, voire leurs armes. 
Outre les thèses fondamentales et les textes introuvables, [...] Slatkine Reprints s'est attachée à réimprimer les grands dictionnaires de la langue française, ainsi que les périodiques des XVII e, XVIII e et XIXe siècles. Son catalogue de plus de 8000 titres, disponible aujourd'hui sur Internet, offre les rééditions indispensables à toute bibliothèque universitaire ou spécialisée en étude française. ${ }^{17}$

Le public visé est ici universitaire, l'intérêt de telles reproductions résidant dans la transmission du contenu du livre et non de sa mise en forme ou plus généralement du geste éditorial qui lui a donné naissance. L'objectif de ces publications est davantage de fournir des rééditions de textes épuisés ou introuvables plutôt que la reproduction d'éditions anciennes. Le fac-similé apparait alors comme un outil de diffusion, au même titre que la reproduction numérique, en ce qu'il n'engage qu'un travail mécanique de reproduction sans renouvellement de l'apparat critique.

Ces diverses entreprises rendent compte de la diversité des fonctions et des usages des fac-similés d'éditions anciennes, suivant le projet éditorial qui préside à leur publication. Si l'articulation du texte à l'ouvrage imprimé semble mise en avant par le choix même de l'impression en fac-similé, on remarque toutefois que ces éditions s'adressent à des publics distincts, s'intéressant en priorité tantôt au texte, tantôt au livre, mais rarement à ce que le livre peut dire du texte et à l'acte de transmission même qu'il opère et qui est pourtant au cœur de son identité.

\section{De l'édition numérique à l'impression à la demande}

Dans le même temps, les avancées technologiques en matière de reproductions numériques ont rendu accessibles à un public de plus en plus divers des éditions «virtuelles» d'œuvres littéraires, dans leurs éditions d'origine. Ce phénomène n'a pas exclu, loin de là, les éditions des œuvres du passé (songeons à l'offre de «classiques» sur Kindle, par exemple). L'accessibilité aux écrits anciens s'est surtout considérablement accrue par la numérisation massive des fonds des grandes bibliothèques publiques, provoquant une relative démocratisation de l'accès aux imprimés anciens ${ }^{18}$. Ces bibliothèques virtuelles, telles Gallica ou les Bibliothèques Virtuelles Humanistes, répondent à une politique éditoriale de valorisation du patrimoine écrit qui vise aussi bien à la préservation des éditions anciennes, moins communiquées du fait de l'existence d'un substitut numérique, qu'à leur

17 Présentation des éditions Slatkine: http://www.slatkine.com/fr/content/11-notre-societe/, consulté le 18 septembre 2016.

18 L'accès à ces éditions est le plus souvent gratuit, mais pas toujours, du fait de leur régime juridique - elles sont libres de droit. Voir la récente polémique à propos du contrat passé entre la BnF et Google. 
diffusion à un public bien plus large que ne le permettait la transmission en salle de lecture.

Certaines d'entre elles proposent depuis peu, aux côtés d'autres ateliers indépendants ${ }^{19}$, la possibilité d'une impression à la demande, en partenariat avec des sociétés d'impression ou des éditeurs traditionnels ${ }^{20}$. Ce développement récent de l'édition numérique du côté de l'impression papier indique bien la situation de transition médiologique où nous nous trouvons: si la culture de l'image favorise un retour à la visualisation des dispositifs de présentation du livre ancien, le développement des écrits d'écran entraîne une forme d'incomplétude que l'impression à la demande voudrait, en vain, venir pallier. L'apparition de ces éditions électroniques témoigne plus globalement du statut ambivalent, à l'heure des écrits d'écran, du traitement des données matérielles : si l'organisation spatiale de la page est bien reproduite à l'identique, ce que rendait possible le fac-similé version papier mais n'autorisait pas l'édition critique traditionnelle, la restitution de l'objet-livre demeure amputée d'une partie de ses principaux constituants: son volume, son poids, les gestes de manipulation qui l'accompagnent, en somme toute une part de son dispositif de signification.

Certaines maisons d'édition font même de la numérisation des documents anciens un commerce qui se présente comme relevant du livre et de l'édition. Ainsi, les éditions NabuPress exploitent l'accès quasi gratuit à des éditions numérisées par les grandes bibliothèques pour assurer ensuite, à titre payant, la reproduction de documents d'époque, éventuellement regroupés par thème. Après avoir apposé une date de copyright, avoir obtenu un nouvel ISBN et avoir créé pour l'occasion une couverture qui constitue en réalité la vitrine d'un travail qui n'a pas été entrepris par la société en question, l'ouvrage est mis en

19 Évoquons l'atelier Librarii, qui se présente, sur la page d'accueil de son site, comme un «atelier de reliure et de restauration du livre, spécialisé également dans la reconstitution des matériaux et techniques picturales traditionnelles. [...] En dehors du travail classique de restauration (papier, reliure) nous proposons des copies de documents : œuvre non restaurable, copie pour exposition, recherche en histoire des techniques ou analyse comparative, copie de sauvegarde, etc. Nous fabriquons les encres, les pigments, les médiums et tous matériaux correspondant aux matériaux originaux puis les mettons en œuvre tel que cela a pu être fait à l'époque, en intégrant les traitements préventifs de conservation » (http://www.librarii.org/, consulté le 18 septembre 2016). Citons également, sur la scène éditoriale française, les éditions du Sonneur, qui publient, sur impression à la demande, «des textes inédits et des textes oubliés ou méconnus dignes de vivre ou de revivre, d'être découverts ou retrouvés ». Mentionnons enfin, pour illustrer un lien étroit entre la publication numérique et l'édition papier, le site Publie.papier et notamment sa collection «Classiques et traductions », émanant de la maison d'édition lancée par Fr. Bon, Publie.net, en partenariat avec Hachette Livre pour la distribution.

20 On pense notamment à la collaboration signée en 2011 entre la BnF et la société Édilivre, qui se charge d'effectuer la reproduction à la demande des ouvrages qui figurent dans le catalogue numérique Gallica et les insère dans sa collection « Livres du Patrimoine ». 
vente sur internet et ne sera imprimé que lorsqu'il aura été commandé et payéế La polémique autour de ces sociétés, qui pratiquent ce que certains appellent la « copyfraud 22 », témoigne de la dissociation qui est en train de s'opèrer entre le texte et le livre, comme si la propriété intellectuelle ne concernait que le seul texte et que ceux qui en assuraient la médiation, y compris numérique, ne disposaient pas de la moindre forme de paternité.

Un tel phénomène engage une vraie réflexion sur la définition de l'acte même d'édition : l'impression à la demande vise-t-elle encore à produire des livres? publier un livre, dans un tel cas, ne se réduit-il pas alors à un simple moyen de reproduction, certes rapide, efficace et peu coûteux, mais dont les usages, mise à part l'accessibilité quasi immédiate du texte, sont finalement assez réduits? et sur ces divers supports, que le tirage ait lieu ou non, que reste-t-il de l'œuvre? à quoi bon restituer la présentation matérielle de l'édition ancienne si le support ne s'accompagne pas d'un dispositif susceptible d'en livrer l'intelligibilité ?

Le cas radical de l'impression à la demande, forme de degré zéro du facsimilé, souligne l'importance de la valeur symbolique de la matérialité du facsimilé dans la transmission des œuvres anciennes : le fac-similé en version papier reste un livre, non pas seulement parce qu'il en adopte la forme habituelle, celle du codex, mais parce qu'il participe d'un réseau de production, de distribution et d'accès au livre inscrit dans une culture de l'imprimé qui concourt à la médiation des œuvres. Aussi, la présence, sur la table d'une librairie, du facsimilé d'une œuvre ancienne à côté d'autres éditions modernes constitue une invitation à manipuler l'ouvrage, à le feuilleter dans sa matière, à le conserver avec soi de manière à ce qu'il habite notre quotidien, ce que ne peut faire un fichier numérique. En cela, le fac-similé d'édition ancienne fait signe vers le livre original dont il est la reproduction, il permet d'en restituer une forme de présence au monde qui fait partie de l'essence du livre et par là même, s'inscrit dans une poïétique, dans un faire, qui nécessite l'investissement corporel du lecteur.

Ce retour à la matière, à la mobilité du texte mis en belle forme de livre, n'exclut pas, bien au contraire, les entreprises engagées de nos jours par les humanités numériques, sans doute les plus à même de donner à lire, par les outils de recontextualisation visuelle adéquats, la matérialité du livre ancien. Les sites donnant accès à l'œuvre ancienne dans ses diverses versions, associant la reproduction de l'image et la transcription diplomatique du texte, comme le propose le programme des Bibliothèques Virtuelles Humanistes, peuvent

21 À titre d'exemple, citons les ouvrages suivants, qui reprennent des ouvrages publiés au $\mathrm{XIX}^{\mathrm{e}}$ siècle comprenant déjà des fac-similés : F. Rabelais à la faculté de Médecine de Montpellier : autographes, documents et fac-simile; Les Songes drolatiques de Pantagruel, où sont contenues cent vingt figures de l'invention de Maistre François Rabelais: copies en fac-simile; Documents inédits sur F.-B. Poquelin Molière : avec des notes, un index alphabétique et des fac-simile.

22 J. Mazzone, « Copyfraud », New York University Law Review, vol. 81, n 3, 2006, p. 1026. 
également proposer au lecteur de multiples documents d'accompagnement, comme le fait par exemple le site Artamène ${ }^{23}$. Dans ces diverses réalisations numériques, il s'agit de faire participer le fac-similé tout autant à la restitution d'une authenticité de l'œuvre passée qu'à son investigation moderne, à la lumière des préoccupations qui sont aujourd'hui les nôtres.

Soulignons en conclusion la grande diversité de l'offre éditoriale en matière de fac-similés aujourd'hui. Le dispositif de reproduction en fac-similé permet le passage d'un support de l'écrit à l'autre, du manuscrit à l'imprimé jusqu'aux écrits d'écran. L'intérêt de ces diverses éditions est qu'elles coexistent et touchent dans le même temps des publics communs et divers à la fois. Cette actualité éditoriale témoigne d'un goût pour la matérialité du livre, relevant de l'affectif, engageant plus une manipulation du livre qu'une lecture du texte. Elle confirme le rôle de l'imaginaire et du symbolique dans la valeur que nous accordons à l'objet-livre et l'importance, encore de nos jours, de posséder le livre dans sa bibliothèque, comme un objet conservé à portée de main. Les nouvelles techniques de reproduction du livre ancien, en version papier et sur écran, loin de figer le livre dans l'image d'un bel objet voué à la seule commémoration, rendent aussi possible une réflexion nouvelle sur le texte mis en mouvement à la lumière de ses supports et sur l'acte d'édition comme acte d'interprétation. Elles viennent alors renouveler l'intelligibilité d'une matérialité du livre qui risquait de ne devenir qu'ornementale, confirmant le célèbre propos de McLuhan : «the medium is the message ${ }^{24} »$.

\author{
Anne Réach-Ngô \\ Université de Haute-Alsace \\ Institut Universitaire de France
}

23 http://www.artamene.org/ (consulté le 18 septembre 2016).

24 M. McLuhan, Understanding Media: The Extensions of Man, New York, McGraw-Hill, 1964 : « Le message, c'est le medium » (titre du premier chapitre). 